\title{
Whither Oxide Electronics?
}

\section{Ramamoorthy Ramesh and Darrell G. Schlom, Guest Editors}

\begin{abstract}
The theme of this issue on "Whither Oxide Electronics?" is an outgrowth of the 2007 David Turnbull Lecture, presented by Ramamoorthy Ramesh, on November 27, 2007, at the 2007 Materials Research Society Fall Meeting in Boston. The Lectureship is awarded annually by MRS to recognize the career of a scientist who has made outstanding contributions to understanding materials phenomena and properties through research, writing, and lecturing, as exemplified by David Turnbull of Harvard University.
\end{abstract}

\begin{abstract}
In this issue we have endeavored to answer the question, "Whither oxide electronics?" This issue provides a framework and perspective on the progress in the field of oxide electronics over the past several decades, as well as the challenges and opportunities in the years to come. Building on the foundations laid by the pioneers in the materials community and spurred by the discovery of high-temperature superconductivity, there has been both tremendous progress in understanding the complex science of oxide electronic materials and the discovery of other fascinating new phenomena, including colossal magnetoresistance, multiferrocity, and twodimensional electron gases in correlated oxide systems. Thin-film heterostructures provide a pathway to create novel devices and combinations of physical phenomena. Indeed, the ability to synthesize and control oxide heterostructures using sophisticated deposition techniques has become a key enabler of the recent advances in this field. These oxides are beginning to enter mainstream products because of their higher performance, for example, ferroelectric memories and oxides with high dielectric constant for computers that run at higher speed and use less power.
\end{abstract}

\section{A Brief History of Oxide Electronics}

The field of complex oxides traces its lineage to the pioneering work of Sir Neville Mott ${ }^{1}$ and co-workers, who originally identified the complex nature of the electronic behavior of oxides, even those as simple as $\mathrm{NiO}$. Their work illuminated the role of electron correlations in determining the transport properties of such materials: correlated electron effects. In the 1940s, the wartime effort demanded new and better materials for a broad range of applications and stimulated Arthur von Hippel's research ${ }^{2}$ on dielectric perovskites and related materials. In the 1950s, the work of Goodenough $^{3}$ and his generation led to some dramatic developments in understanding the crystal chemistry and transport physics of doped transition-metal perovskites. This work especially focused on the manganites, which, in essence, revisited the correlated behavior of electrons in the broad family of perovskites. Indeed, many of the "rules" of interactions in these transition-metal-based oxides were outlined in this period. In parallel, the work of Eric Cross and colleagues ${ }^{4}$ on perovskite dielectrics both opened up the field of technologically viable ferroelectrics and piezoelectrics and established a strong fundamental scientific basis for these materials. During these decades, Ted Geballe ${ }^{5}$ played a significant role in the discovery of advanced materials of all sorts, including oxides. Finally, the field of complex oxides would not be where it is now without the seminal contributions of K. Alex Müller, ${ }^{6}$ Georg Bednorz, ${ }^{6}$ and Bernard Raveau in the area of superconductivity. This "fast forwards" us to the 1980s and to hightemperature superconductivity.

The fascinating properties of complex oxides continue to infatuate and drive materials scientists to master both understanding and synthesis of the materials, with the goal of exploiting these properties in devices. In addition to superconductivity, the properties include ferromagnetism (materials where an applied magnetic field can cause the spontaneous magnetization of the material to flip) and ferroelectricity (materials where an applied electric field can cause the spontaneous polarization of the material to flip), and even materials that are both at the same time. The crystal structures of several of the key structural families of complex oxides are shown in Figure 1.7

\section{The Impetus of High-Temperature Superconductivity}

The advent of superconductivity in

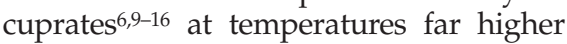
than any previously known superconductors (making them "high-temperature" superconductors) has been, by far, the most significant development in the field of condensed matter sciences in the past several decades. It brought the field of complex oxides onto the main stage. The potential for applications of these materials in next-generation Josephson junctions for high-speed, low-power computing, sensitive magnetic field sensors, and highfrequency microwave filters stimulated the evolution and implementation of a variety of thin-film deposition techniques.

Venkatesan and co-workers pioneered the synthesis of complex oxides by pulsed-laser deposition. ${ }^{17}$ This is a novel approach to create stoichiometric films and layered stacks of different complex oxides (heterostructures). The dynamic interaction of the material with short ultraviolet laser pulses circumvents the differences in vapor pressures of the constituents. The ability to flash evaporate the surface of a target enables this singletarget method to produce films with nearly the same composition as the multicomponent target from which they are made. Molecular-beam epitaxy, a key tool that enabled the growth of semiconductor films following the crystalline template of the single-crystal substrate beneath them (semiconductor heteroepitaxy) in the 1970s and 1980s ${ }^{66}$, was also employed to grow these multicomponent oxides. ${ }^{18-20}$ In parallel, electron-beam evaporation,,21,22 sputtering, ${ }^{23-27}$ and ion-beam deposition ${ }^{28}$ evolved as viable physical vapor deposition techniques for complex oxides, and a strong effort toward the discovery of new organometallic precursors for chemical vapor deposition was initiated. ${ }^{29}$

\section{The Example of Ferroelectric Memories}

While the high-temperature superconductor revolution was emerging, other 


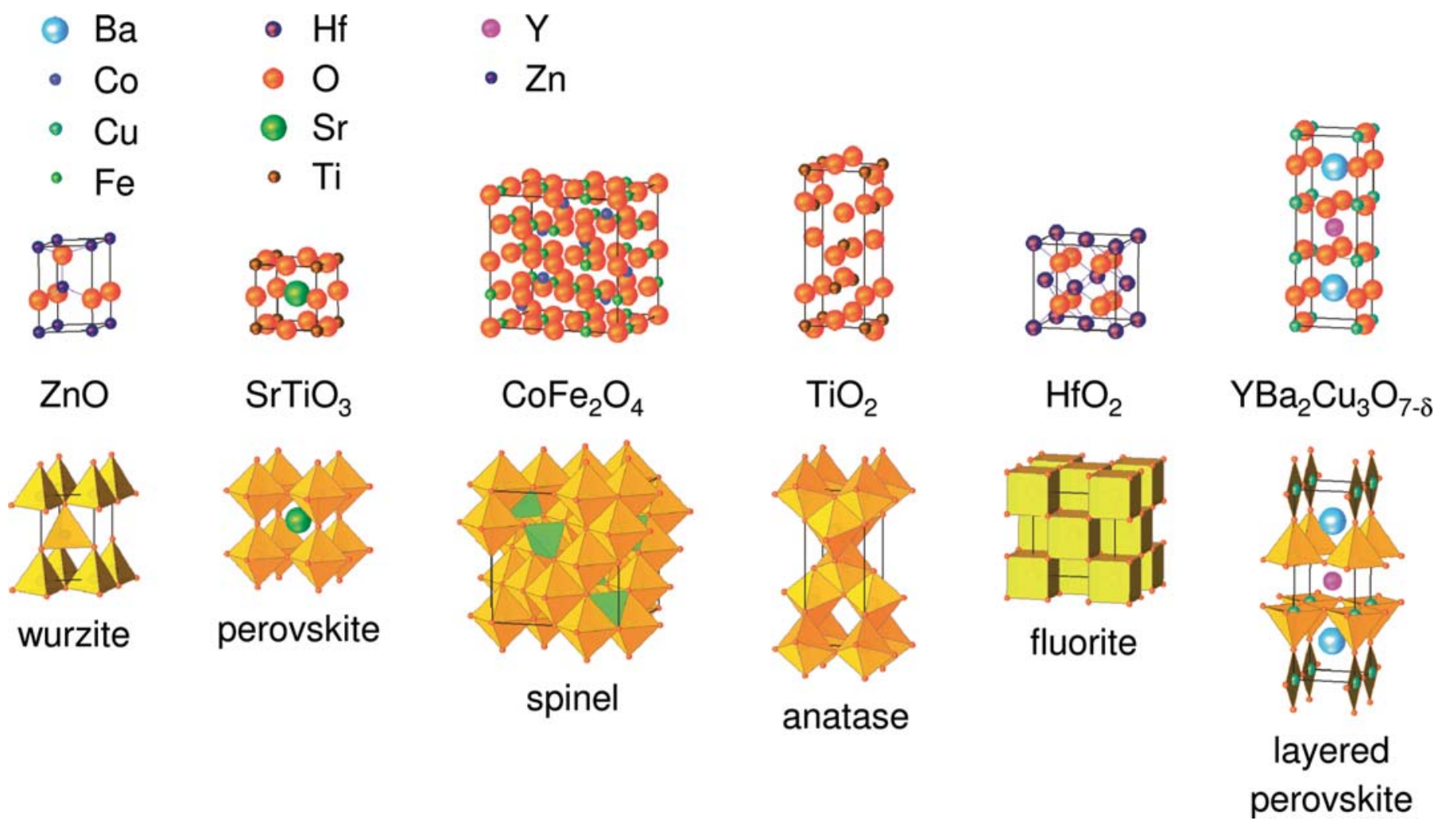

Figure 1. Examples of the crystal structures of complex oxides with striking properties that are the subject of the articles in this issue. Two equivalent representations of these crystal structures are shown: the atomic positions (top) and the coordination polyhedra (bottom). The oxygen atoms occupy the vertices of the coordination polyhedra. Color is used to distinguish the two types of oxygen coordination polyhedra in $\mathrm{CoFe}_{2} \mathrm{O}_{4}$, namely, octahedra (orange) and tetrahedra (green). The relative sizes of the atoms reflect their relative ionic radii as reported in Reference 7.

oxide-related fields were gaining prominence as well. The critical need for a highdensity, solid-state memory technology was the key driver for researchers to explore ferroelectric thin films to store binary bits in the form of the two polarization states of a ferroelectric material. ${ }^{30}$ Of course, the potential of ferroelectrics as memory elements had been recognized in the late 1950 s. ${ }^{31}$

Three key issues limited the pervasive implementation of ferroelectrics in such technologies. First, these materials generally do not exhibit a well-defined switching threshold. Matrix-addressed memory architecture was becoming popular in the 1960s because of the emergence of semiconductor integrated circuit technologies, and in these devices, all of the bits would be subjected to at least one-half of the probing voltage. Under such conditions, the ferroelectrics would exhibit a finite probability of switching their polarization state, that is, the so-called "half-voltage disturb effect." This problem was solved by adding a transistor to each memory cell so that the write voltage could reach the ferroelectric memory element and alter its polarization state only when the transistor is activated.
This pass-transistor architecture is now common in all solid-state memories.

The second issue was the switching voltage itself. Note that, in the 1960s, advanced thin-film deposition techniques for such complex materials were not available. Therefore, much of the work was carried out on bulk crystals, which needed switching voltages of $\sim 100 \mathrm{~V}$. Such high voltages were completely incompatible with the emerging memory technologies based on complementary metal oxide semiconductor (CMOS) materials, all of which used $5 \mathrm{~V}$ operating voltages. Once again, this issue was solved simply by changing to thin films.

By far, the most serious difficulty with implementing thin film-based ferroelectric memories was a fundamental materials issue related to the interactions of ferroelectric domains with the film microstructure, especially defects. This gave rise to two problems. The first of these was fatigue, which is a drop in the polarization with progressive switching. The second related issue was imprint, which is a preference of the ferroelectric storage element to remain in (or go back to) one of the two states. Both of these problems were related to how domains interacted with point defects as well as electronic defects, with the role of the electrode/ferroelectric interface being particularly critical.

In the early 1990s, a few groups began addressing these two issues. They explored conducting perovskites including cuprate superconductors, ${ }^{32-34} \mathrm{SrRuO}_{3}, 34,35$ $(\mathrm{La}, \mathrm{Sr}) \mathrm{CoO}_{3},{ }^{34,36}$ and $(\mathrm{La}, \mathrm{Sr}) \mathrm{MnO}_{3}{ }^{37} \mathrm{In}$ parallel, Kingon et al., ${ }^{38}$ as well as researchers at National Semiconductor, were exploring $\mathrm{RuO}_{2}$, again a conducting oxide electrode material. In early 1991, both oxide-based approaches proved to be successful in solving the problem of fatigue. The key breakthrough was the recognition that charged oxygen vacancies (and, to a smaller extent, electronic defects) could segregate to the interface between the electrode (typically $\mathrm{Pt}$ ) and the ferroelectric lead zirconate titanate (PZT) film, causing a space-charge layer that subsequently prevented switching; indeed, a similar defect-mediated mechanism is likely to be operative in causing imprint.

Are oxide electrodes the only approach to solving the problem of fatigue? This might not be the case, as the work of Scott 


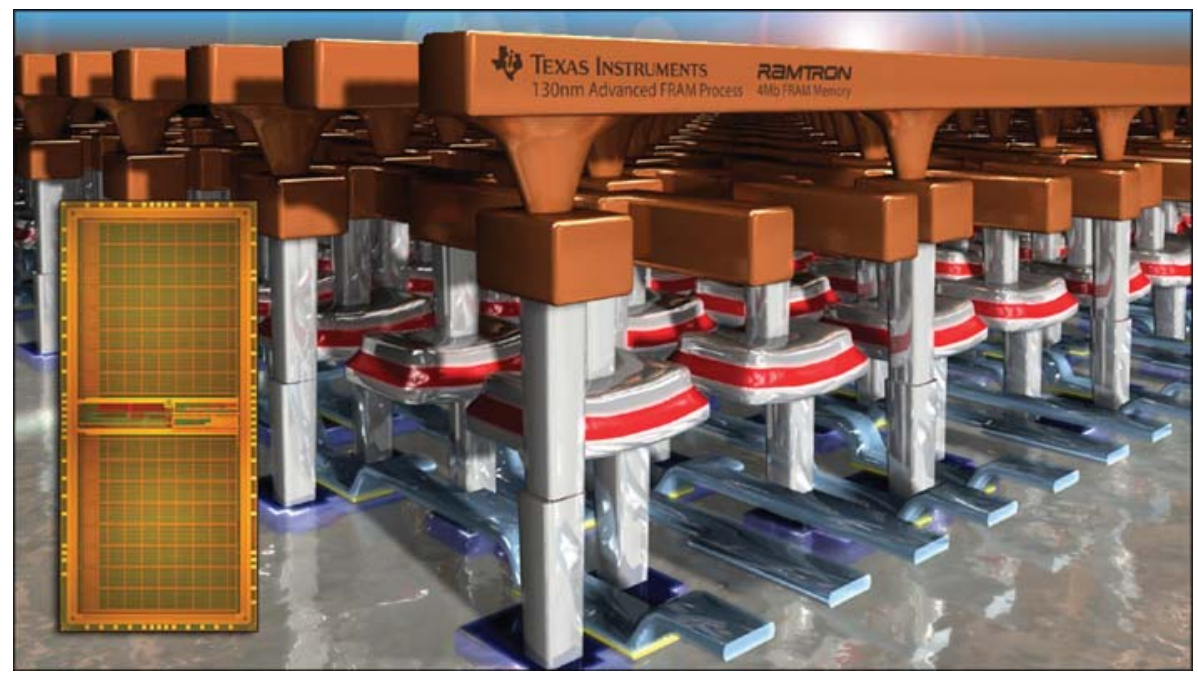

Figure 2. Schematic of the architecture of a 4-Mb ferroelectric random access memory device (from Texas Instruments/Ramtron).

and Araujo showed the possibility of using an alternative ferroelectric, $\mathrm{SrBi}_{2} \mathrm{Ta}_{2} \mathrm{O}_{9}$ with $\mathrm{Pt}$ electrodes without any significant fatigue. ${ }^{39}$ Thus, the electrode/ferroelectric interface is important, but the domain dynamics within the framework of defect interactions is also critical.

Translating these basic materials science discoveries into a manufacturable, commercial technology requires several steps and materials innovations. For example, these ferroelectric oxide capacitor stacks have to be integrated with a conducting plug directly on top of the pass transistor in the one-transistor-one-capacitor $(1 \mathrm{~T}-1 \mathrm{C})$ cell of advanced ferroelectric memories. ${ }^{40}$ Such a cell, which is currently being manufactured by Texas Instruments, ${ }^{40}$ is shown in the image in Figure 2. The ferroelectric capacitor stack must be deposited by a scalable process suited to manufacturing, such as chemical vapor deposition or sputtering.

The capacitors also have to be defined through a dry etch process, another critical step. After the process is completed, the memory cell must be treated with forming gas $\left(\mathrm{H}_{2} / \mathrm{N}_{2}\right.$ mixture) to clean up traps at the transistor interface. This step can destroy the ferroelectric oxide, and therefore, a robust passivation layer is needed. It is satisfying to note that the field has successfully solved all of these integration and reliability problems, thus paving the way to real, high-density memories. Several companies (such as Texas Instruments, Fujitsu, Toshiba, Panasonic, and Sony) are in the advanced stages of product development of high-density, embedded ferroelectric memories. This could be the emergence of the so-called "universal memory."

\section{Renaissance of Correlated Electron Materials}

The early 1990s witnessed the "rebirth" of correlated electron oxides, in this case through the manganites. High-temperature superconductors had already set the stage for this rebirth by providing both the intellectual framework and the experimental and theoretical tools to explore related systems. The work of Chahara et al. ${ }^{41}$ and von Helmolt et al. ${ }^{42}$ triggered the work of Jin and co-workers ${ }^{43}$ in the pursuit of materials that exhibit dramatically large (dubbed "colossal") changes in transport properties upon the application of a magnetic field. ${ }^{16}$ These early works attracted much attention around the world, setting the stage for an explosion of research activity that is yet to abate.

A model system of correlated oxides is the antiferromagnetic insulator $\mathrm{LaMnO}_{3}$. Upon doping with holes (e.g., Sr substitution in the La site), the system becomes electronically inhomogeneous and is highly susceptible to perturbations and external manipulation. ${ }^{44-46}$ As a consequence, the phase diagram of such a system (Figure 3) exhibits multiple phases that are susceptible to change through temperature; chemical doping; and electric, magnetic, and mechanical fields. The article by Dagotto and Tokura in this issue details the properties of these extraordinary materials.

A key aspect to keep in mind is the energy scale involved in creating these changes, as it directly impacts the ability to translate these discoveries into commercial technologies. For example, the colossal magnetoresistance (CMR) effect, the change in resistance accompanying the application of a magnetic field, is typically observed

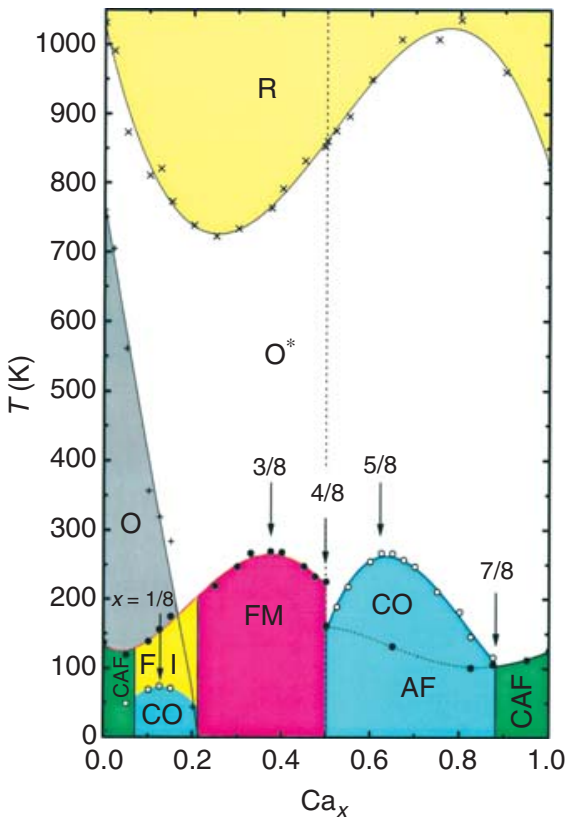

Figure 3. Phase diagram of hole-doped manganites. The parent structure is an antiferromagnetic insulator, but with doping, it becomes ferromagnetic and exhibits the colossal magnetoresistance effect. Analogous phase diagrams occur in high- $T_{c}$ superconductor and relaxor ferroelectric systems. Note: AF is antiferromagnetic, $\mathrm{FM}$ is ferromagnetic, $\mathrm{CAF}$ is canted antiferromagnetic, $\mathrm{CO}$ is charge-ordered, $\mathrm{FI}$ is ferromagnetic insulating, $\mathrm{R}$ is rhombohedral, $\mathrm{O}$ is orthorhombic (Jahn-Teller-distorted), and $\mathrm{O}^{*}$ is orthorhombic (octahedron rotated). (Reprinted from Reference 67 with permission).

with a magnetic field of a few Tesla (1-10 T). This translates to a temperature scale of $\sim 10$ $\mathrm{K}$, which is certainly a small value compared to the temperatures $(200-380 \mathrm{~K})$ at which the CMR effect peaks. On the other hand, creating such a magnetic field, say, 6 $\mathrm{T}$, requires a current of several amperes running through a wire. Clearly, this is technologically prohibitive and is the main reason that products, such as magnetic sensors and read heads, involving CMR materials have not emerged.

This difficulty also provides some insight into exploring new materials in the future: If magnetic fields are to be used, then they have to be competitive with current technologies, such as giant magnetoresistance (GMR)-based magnetic tunnel junctions used in the read heads of hard disks. ${ }^{47}$ On the other hand, if electric fields are used, then the large magnetoresistance effects observed at a magnetic field of $6 \mathrm{~T}$ can be obtained at fields on the order of a few hundred kilovolts per 
centimeter, which translates to a potential of a few volts across a 100-nm-thick film. A key conclusion emerges from this analysis: Electric-field-based modulation of properties is much more feasible if thin films and thin film heterostructures are used. This naturally leads us to the field of multiferroics and magnetoelectrics, which is described in more detail in the article by Spaldin and Ramesh in this issue.

\section{Atomic-Scale Engineering: A Key Enabler of Oxide Electronics}

Having started with vacuum deposition tools that have achieved unparalleled control in the integration of semiconductors at the nanometer level, it should come as no surprise that these same tools, once properly modified for the challenges of oxides, are now producing customized oxide heterostructures with control at the atomic level. ${ }^{48-53}$ These epitaxial techniques allow for the preparation of ultrathin oxides that can be used for a range of purposes: studying the thickness at which ferroelectricity and other functional properties emerge, ${ }^{54,55}$ determining ultimate scaling limits, ${ }^{54-56}$ orienting oxides in directions that maximize a desired property, creating metastable oxides, integrating complex oxides with conventional semiconductors, ${ }^{57}$ synthesizing oxide heterostructures containing electrodes and multifunctional active layers all in an epitaxial stack, and making oxide superlattices. ${ }^{48-52}$ Even single interfaces can have surprising properties, for example, the conductive (and even superconducting) interface between the two insulators $\mathrm{SrTiO}_{3}$ and $\mathrm{LaAlO}_{3}$. See the article by Mannhart, Blank, Hwang, Millis, and Triscone in this issue for a full discussion of two-dimensional electron gases at interfaces.

An example of an oxide superlattice is shown in Figure $4 a .{ }^{58}$ The perovskites $\mathrm{SrTiO}_{3}$ and $\mathrm{BaTiO}_{3}$ are alternately deposited to build up a 2-2 epitaxial composite, in which both constituents of the composite are connected in two dimensions. ${ }^{59}$ Although $\mathrm{SrTiO}_{3}$ and $\mathrm{BaTiO}_{3}$ are fully miscible and form a complete solid solution in the bulk, ${ }^{60}$ bulk diffusion is minimal at the low substrate temperature involved in oxide epitaxy (typically $700^{\circ} \mathrm{C}$ ), and such atomically abrupt metastable heterostructures are readily grown. Such superlattices not only are relevant to fundamental science involving size, strain, and the coupling of order parameters between constituent materials, but are also being investigated for novel applications including acoustic cavities for terahertz modulators or phonon lasers. ${ }^{61} 1-3$ epitaxial composites, ${ }^{59}$ where one composite constituent is connected in just one dimension and the other is connected in three dimen-
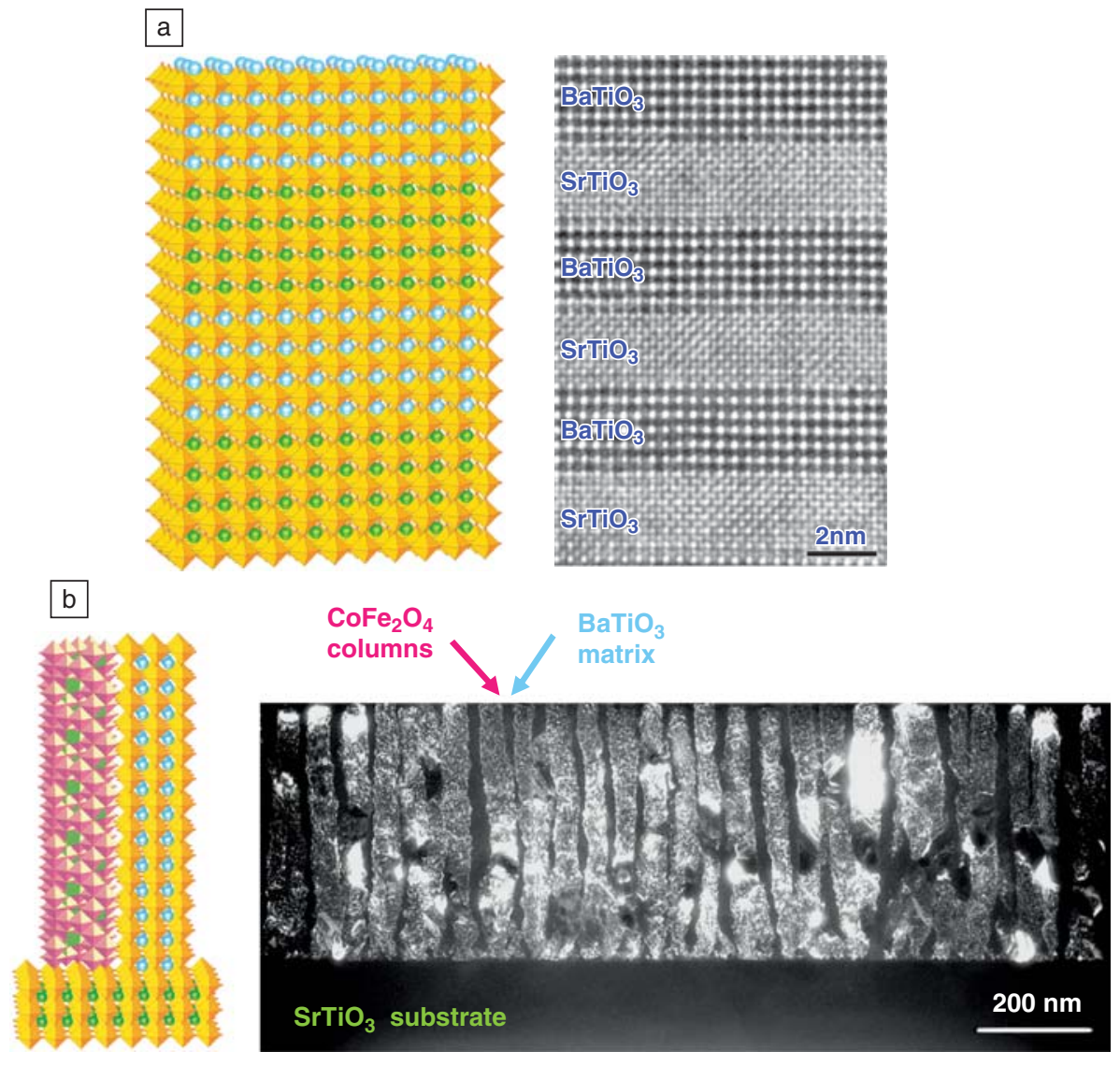

Figure 4. Examples of (a) layered epitaxial heterostructures (2-2 composites) and (b) epitaxial 1-3 nanocomposites produced by modern complex oxide growth methods. A transmission electron microscopy (TEM) image of each type is shown together with a schematic. (Figure 4a is reprinted from Reference 58 with permission. Copyright 2006, American Institute of Physics. Figure 4b is reprinted from Reference 62 with permission. Copyright 2004 American Institute of Physics.)

sions, are also possible, and such composites with pillar dimensions as small as 20 $\mathrm{nm}$ have been demonstrated, ${ }^{62}$ as shown in Figure $4 \mathrm{~b}$.

Because epitaxy is involved, huge (percent-level) strains can be imposed on oxide films. With sufficiently defect-free substrates, the crystalline perfection of heteroepitaxial films of complex oxides can rival that of single crystals of the same composition. ${ }^{63}$ Finally, these same epitaxial oxides can be doped to create new functionalities. The article by Kamiya and Kawasaki in this issue addresses the topic of doping $\mathrm{ZnO}$ to make it magnetic and optically active, and the article by Coey and Chambers in this issue explores the controversial topic of magnetism in doped $\mathrm{TiO}_{2}$ films.

Nonepitaxial heterostructures are also of interest, with a key recent technological breakthrough involving the replacement of the $\mathrm{SiO}_{2}$-based gate dielectric in computer chips with a $\mathrm{HfO}_{2}$-based alternative. Here, the heterostructures are amorphous and polycrystalline. The higher dielectric constant of this alternative gate dielectric, while maintaining the excellent electrical characteristics that made the $\mathrm{SiO}_{2} / \mathrm{Si}$ interface the material of choice for metal oxide semiconductor field-effect transistors, provides for higher performance computers that operate at higher speeds and lower power. Its development is enabling transistor scaling (Moore's law) to continue for at least a few more years. Achieving this materials breakthrough involved overcoming a multitude of materials issues as described in the article by Schlom, Guha, and Datta in this issue.

\section{The Future}

So, what does the future of oxide electronics hold? We believe we are at the cusp of some dramatic developments in the science of complex oxides, especially in the form of thin-film heterostructures. At the fundamental level, the novel electronic phenomena that exist in superconducting cuprates and CMR manganites 
can be summarized into a rather simple theme: It is doping a well-behaved antiferromagnetic insulator with carriers (holes or electrons) or creating local inhomogeneities in charge distribution that leads to such physically interesting behavior as a consequence of a two-phase coexistence.

The next question is this: Can we employ this approach as a generic algorithm to explore other novel phenomena that occur at the phase boundaries and tap into phenomena that involve interactions between electron spin, electron charge, electron orbitals, and the underlying atomic structure of a material? For example, what happens if one dopes a multiferroic (which has both an antiferromagnetic and another order parameter)? What are the appropriate length and energy scales for doing so? Clearly, significant improvements in synthesis, especially in the control of chemical stoichiometry through the implementation of complementary surface analytical tools (e.g., electron diffraction coupled with simultaneous surface composition measurement through analysis of emitted characteristic x-rays ${ }^{64}$ ), will play a critical role in enabling us to probe such novel phenomena.

A major challenge for the field is control over oxygen stoichiometry. Indeed, this could be one of the most significant opportunities for the future. How does one control oxygen in the way that our semiconductor colleagues control dopants and impurities in $\mathrm{Si}$ and GaAs? Can one quantitatively determine and control the surface termination of oxide heterostructures during growth?

\section{Harnessing Novel Properties for Potential Devices}

An important future direction that will be particularly stimulated by the multiple functionalities in complex oxides is crosscoupling or decoupling of fundamental phenomena. Magnetoelectric and multiferroic effects provide one such example that deals with the coupling between electric and magnetic degrees of freedom. This is but the beginning. As we forge ahead, we need to keep in mind the fact that both science and technology must work together, as illustrated in Figure 5. We need to be cognizant of both the competition (at the technology and market level) and the critical issues that can limit the transition of basic science into technology.

Complex oxides are poised to play a key role in the rapidly emerging area of materials for energy technologies. One can envision creating oxide heterostructures and nanostructures that will enable the decoupling of electron and phonon transport, of particular relevance to the design of next-generation thermoelectrics.

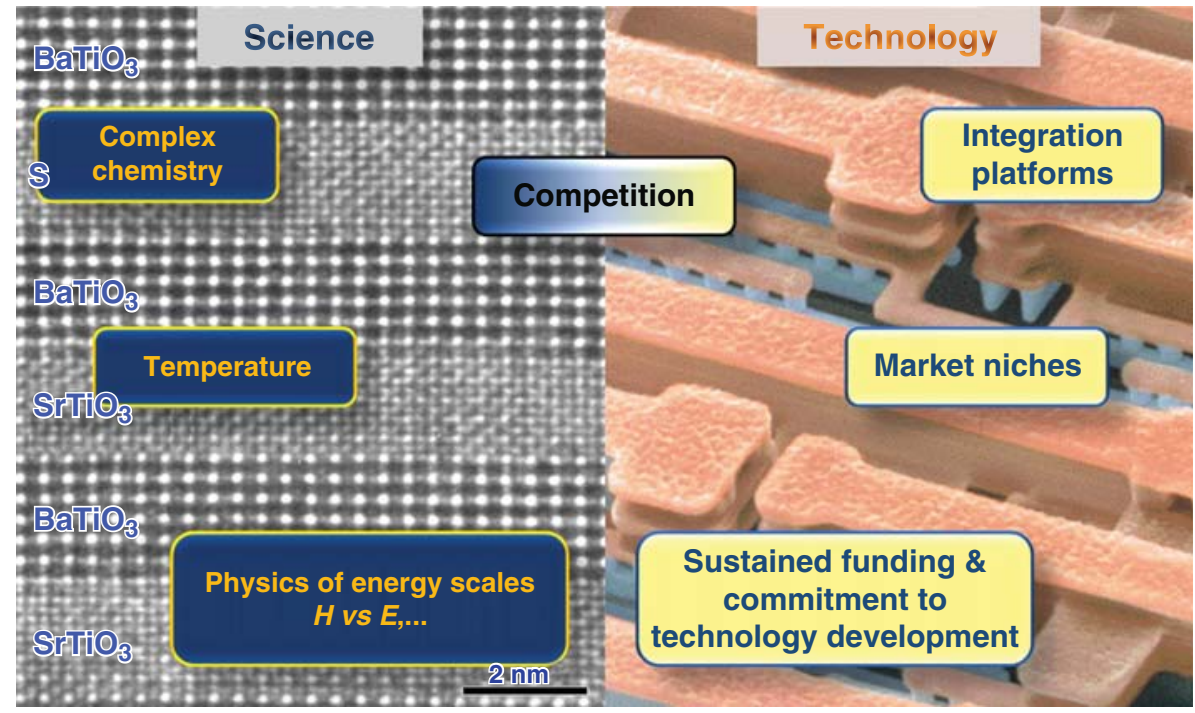

Figure 5. Illustration of the interplay between basic science and technology development. On the left side are shown some of the critical issues that need to be addressed at the fundamental level. On the right side are shown the factors that determine technology creation and commercialization. It is also clear that sustained resource commitment to both components is crucial for successful introduction of new fundamental discoveries into the technology stream. (The background of the left panel is reprinted from Reference 58 with permission. Copyright 2006 American Institute of Physics. The background of the right panel is courtesy of International Business Machines Corporation. Unauthorized use is not permitted.)

The ability to engineer artificial heterostructures (e.g., $\mathrm{SrTiO}_{3} / \mathrm{LaAlO}_{3}$ ) with tunable dielectric contrast as well as phonon characteristics is an attractive approach to creating phonon confinement and eventually phonon lasers. Indeed, ongoing work has already demonstrated the rudiments of these possibilities. ${ }^{61,65}$ Another area of research is the conversion of photons to electrons, critical to photovoltaic solar energy conversion and photocatalysis for water splitting and $\mathrm{CO}_{2}$ conversion to usable fuel. The multitude of exciting opportunities for oxides and especially oxide heterostructures will continue to inspire future generations of oxide electricians for decades to come.

\section{Acknowledgments}

First and foremost, we are grateful to the Materials Research Society for the Turnbull Award, which provided us with the rationale for this special issue. We are particularly grateful to our colleagues who agreed to contribute the articles contained in this issue. We are equally thankful to all of our oxide electronics colleagues from around the world who have contributed in many ways to the burgeoning field of oxide electronics. Given the size of this field, it would be impossible to enumerate them individually. The support of funding agencies, within the United States and around the world for research and development support of oxide electronics is critical, and we are extremely grateful for this. Specifically, R. Ramesh acknowledges the support of the U.S. Department of Energy (J. Horwitz and H. Kung) for its enthusiastic support of oxide electronics. The early work on ferroelectrics was stimulated by the vision of Jane Alexander (who was at that time at DARPA).

\section{References}

1. N.F. Mott, Metal-Insulator Transitions (Taylor \& Francis, London, ed. 2, 1990)

2. A. von Hippel, R.G. Breckenridge, F.G Chesley, L. Tisza, Ind. Eng. Chem. 38, 1097 (1946) 3. J.B. Goodenough, in Progress in Solid State Chemistry, H. Reiss, Ed. (Pergamon Press, Oxford, 1971), vol. 5, pp. 145-399.

4. L.E. Cross, Ferroelectrics 76, 241 (1987)

5. T.H. Geballe, MRS Bull. 17 (7), 70 (1992).

6. J.G. Bednorz, K.A. Müller, Z. Phys. B Condens. Matter 64, 189 (1986).

7. R.D. Shannon, Acta Crystallogr. A 32, 751 (1976).

8. R.M. Hazen, Sci. Am. 258, 52 (1988).

9. J.B. Goodenough, G. Demazeau, M. Pouchard, P. Hagenmuller, J. Solid State Chem. 8, 325 (1973).

10. C. Michel, B. Raveau, Rev. Chim. Miner. 21, 407 (1984).

11. J.G. Bednorz, M. Takashige, K.A. Müller, Europhys. Lett. 3, 379 (1987).

12. M.K. Wu, J.R. Ashburn, C.J. Torng, P.H. Hor, R.L. Meng, L. Gao, Z.J. Huang, Y.Q. Wang, C.W. Chu, Phys. Rev. Lett. 58, 908 (1987). 13. R.J. Cava, B. Batlogg, R.B. van Dover, D.W. Murphy, S. Sunshine, T. Siegrist, J.P. Remeika, E.A. Rietman, S. Zahurak, G.P. Espinosa, Phys. Rev. Lett. 58, 408 (1987). 
14. H. Takagi, S.-I. Uchida, K. Kitazawa, S. Tanaka, Jpn. J. Appl. Phys., Part 2 26, L123 (1987). 15. Y. Tokura, H. Takagi, S. Uchida, Nature 337, 345 (1989).

16. M. Imada, A. Fujimori, Y. Tokura, Rev. Mod. Phys. 70, 1039 (1998).

17. D. Dijkkamp, T. Venkatesan, X.D. Wu, S.A. Shaheen, N. Jisrawi, Y.H. Min-Lee, W.L. McLean, M. Croft, Appl. Phys. Lett. 51, 619 (1987).

18. R.J. Spah, H.F. Hess, H.L. Stormer, A.E. White, K.T. Short, Appl. Phys. Lett. 53, 441 (1988). 19. D.G. Schlom, J.N. Eckstein, E.S. Hellman, S.K. Streiffer, J.S. Harris, Jr., M.R. Beasley, J.C. Bravman, T.H. Geballe, C. Webb, K.E. von Dessonneck, F. Turner, Appl. Phys. Lett. 53, 1660 (1988).

20. J. Kwo, M. Hong, D.J. Trevor, R.M. Fleming, A.E. White, R.C. Farrow, A.R. Kortan, K.T. Short, Appl. Phys. Lett. 53, 2683 (1988).

21. D.K. Lathrop, S.E. Russek, R.A. Buhrman, Appl. Phys. Lett. 51, 1554 (1987).

22. T. Terashima, K. Iijima, K. Yamamoto, Y. Bando, H. Mazaki, Jpn. J. Appl. Phys., Part 2 27, L91 (1988).

23. H. Koinuma, M. Kawasaki, M. Funabashi, T. Hasegawa, K. Kishio, K. Kitazawa, K. Fueki, S. Nagata, J. Appl. Phys. 62, 1524 (1987).

24. U. Poppe, J. Schubert, R.R. Arons, W. Evers, C.H. Freiburg, W. Reichert, K. Schmidt, W. Sybertz, K. Urban, Solid State Commun. 66, 661 (1988)

25. R.L. Sandstrom, W.J. Gallagher, T.R. Dinger, R.H. Koch, R.B. Laibowitz, A.W. Kleinsasser, R.J. Gambino, B. Bumble, M.F. Chisholm, Appl. Phys. Lett. 53, 444 (1988).

26. X.X. Xi, G. Linker, O. Meyer, E. Nold, B. Obst, F. Ratzel, R. Smithey, B. Strehlau, F. Weschenfelder, J. Geerk, Z. Phys. B: Condens. Matter 74, 13 (1989).

27. C.B. Eom, J.Z. Sun, K. Yamamoto, A.F. Marshall, K.E. Luther, T.H. Geballe, S.S. Laderman, Appl. Phys. Lett. 55, 595 (1989).

28. J. Fujita, T. Yoshitake, A. Kamijo, T. Satoh, H. Igarashi, J. Appl. Phys. 64, 1292 (1988).

29. D.L. Schulz, T.J. Marks, Adv. Mater. 6, 719 (1994).

30. J.F. Scott, C.A. Paz de Araujo, Science 246, 1400 (1989).

31. J.A. Morton, "Electrical Switching and Storage," U.S. Patent 2,791,761 (May 7, 1957).

32. R. Ramesh, A. Inam, W.K. Chan, B. Wilkens, K. Myers, K. Remschnig, D.L. Hart, J.M. Tarascon, Science 252, 944 (1991).
33. R. Ramesh, W.K. Chan, B. Wilkens, H. Gilchrist, T. Sands, J.M. Tarascon, V.G. Keramidas, D.K. Fork, J. Lee, A. Safari, Appl. Phys. Lett. 61, 1537 (1992).

34. R. Ramesh, "Cubic Metal Oxide Thin Film Epitaxially Grown on Silicon," U.S. Patent 5,270,298 (December 14, 1993).

35. C.B. Eom, R.J. Cava, R.M. Fleming, J.M. Phillips, R.B. van Dover, J.H. Marshall, J.W.P. Hsu, J.J. Krajewski, W.F. Peck, Jr., Science 258, 1766 (1992).

36. J.F.M. Cillessen, R.M. Wolf, A.E.M. De Vierman, Appl. Surf. Sci. 69, 212 (1993).

37. S. Matthews, R. Ramesh, T. Venkatesan, J. Benedetto, Science 276, 238 (1997).

38. H.N. Al-Shareef, A.I. Kingon, X. Chen, K.R. Bellur, O. Auciello, J. Mater. Res. 9, 2968 (1994). 39. C.A-Paz de Araujo, J.D. Cuchiaro, L.D. McMillan, M.C. Scott, J.F. Scott, Nature 374, 627 (1995).

40. T.S. Moise, S.R. Summerfelt, H. McAdams, S. Aggarwal, K.R. Udayakumar, F. Celii, S. Martin, G. Xing, L. Hall, K.J. Taylor, T. Hurd, J. Rodriguez, K. Remack, M.D. Khan, G. Stacey, M. Yao, G. Albrecht, E. Zielinski, B. McKee, J. Rickes, J. Grace, J. Fong, D. Lee, C. Pietrzyk, R. Lanham, S. Gilbert, D. Taylor, J. Amano, R. Bailey, F. Chu, G. Fox, S. Sun, T. Davenport, in IEDM '02 Technical Digest (IEEE, Piscataway, NJ, 2002), pp. 535-538.

41. K. Chahara, T. Ohno, M. Kasai, Y. Kozono, Appl. Phys. Lett. 63, 1990 (1993).

42. R. von Helmolt, J. Wecker, B. Holzapfel, L. Schultz, K. Samwer, Phys. Rev. Lett. 71, 2331 (1993).

43. S. Jin, T.H. Tiefel, M. McCormack, R.A. Fastnacht, R. Ramesh, L.H. Chen, Science 264, 413 (1994).

44. E.L. Nagaev, Phys. Status Solidi B 186, 9 (1994). 45. M. Uehara, S. Mori, C.H. Chen, S.W. Cheong, Nature 399, 560 (1999).

46. E. Dagotto, Nanoscale Phase Separation and Colossal Magnetoresistance: The Physics of Manganites and Related Compounds (Springer, Berlin, 2003).

47. S.S.P. Parkin, Annu. Rev. Mater. Sci. 25, 357 (1995).

48. J.-M. Triscone, M.G. Karkut, L. Antognazza, O. Brunner, Ø. Fischer, Phys. Rev. Lett. 63, 1016 (1989).

49. K. Iijima, T. Terashima, Y. Bando, K. Kamigaki, H. Terauchi, J. Appl. Phys. 72, 2840 (1992).
50. J. Eckstein, I. Bozovic, Annu. Rev. Mater. Sci. 25, 679 (1995).

51. H.-M. Christen, L.A. Boatner, J.D. Budai, M.F. Chisholm, L.A. Géa, P.J. Marrero, D.P. Norton, Appl. Phys. Lett. 68, 1488 (1996).

52. D.G. Schlom, J.H. Haeni, J. Lettieri, C.D. Theis, W. Tian, J.C. Jiang, X.Q. Pan, Mater. Sci. Eng. B 87, 282 (2001).

53. A. Ohtomo, D.A. Muller, J.L. Grazul, H.Y. Hwang, Nature 419, 378 (2002).

54. T. Tybell, C.H. Ahn, J.-M. Triscone, Appl. Phys. Lett. 75, 856 (1999).

55. D.D. Fong, G.B. Stephenson, S.K. Streiffer, J.A. Eastman, O. Auciello, P.H. Fuoss, C. Thompson, Science 304, 1650 (2004).

56. T. Terashima, K. Shimura, Y. Bando, Y. Matsuda, A. Fujiyama, S. Komiyama, Phys. Rev. Lett. 67, 1362 (1991).

57. S. Matsubara, N. Shohata, M. Mikami, Jpn. J. Appl. Phys. 24, Suppl. 24-3, 10 (1985).

58. W. Tian, J.C. Jiang, X.Q. Pan, J.H. Haeni, Y.L. Li, L.Q. Chen, D.G. Schlom, J.B. Neaton, K.M. Rabe, Q.X. Jia, Appl. Phys. Lett. 89, 092905 (2006). 59. R.E. Newnham, D.P. Skinner, L.E. Cross, Mater. Res. Bull. 13, 525 (1978).

60. E.M. Levin, C.R. Robbins, H.F. McMurdie, Eds., Phase Diagrams for Ceramists (American Ceramic Society, Columbus, OH, 1964), vol. 1, p. 195.

61. A. Soukiassian, W. Tian, D.A. Tenne, X.X. Xi, D.G. Schlom, N.D. Lanzillotti-Kimura, A. Bruchhausen, A. Fainstein, H.P. Sun, X.Q. Pan, A. Cros, A. Cantarero, Appl. Phys. Lett. 90, 042909 (2007)

62. H. Zheng, J. Wang, S.E. Lofland, Z. Ma, L. Mohaddes-Ardabili, T. Zhao, L. SalamancaRiba, S.R. Shinde, S.B. Ogale, F. Bai, D. Viehland, Y. Jia, D.G. Schlom, M. Wuttig, A. Roytburd, R. Ramesh, Science 303, 661 (2004).

63. D.G. Schlom, L.Q. Chen, C.B. Eom, K.M. Rabe, S.K. Streiffer, J.-M. Triscone, Annu. Rev. Mater. Res. 37, 589 (2007).

64. S. Ino, T. Ichikawa, S. Okada, Jpn. J. Appl. Phys. 19, 1451 (1980)

65. H. Ohta, S. Kim, Y. Mune, T. Mizoguchi, K. Nomura, S. Ohta, T. Nomura, Y. Nakanishi, Y. Ikuhara, M. Hirano, H. Hosono, K. Koumoto, Nat. Mater. 6, 129 (2007).

66. A.Y. Cho, MRS Bull. 20, 21 (1995).

67. K.H. Kim, M. Uehara, V. Kiryukhin, S.-W. Cheong, in Colossal Magnetoresistive manganites, T. Chatterjii Ed. (Kluwer Academic, Dordrecht, 2004) pp. 131-206.

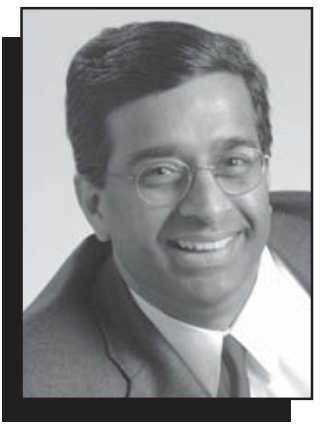

Ramamoorthy Ramesh

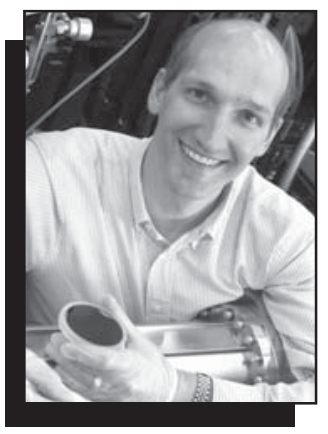

Darrell G. Schlom
Ramamoorthy Ramesh, Guest Editor for this issue of MRS Bulletin, can be reached by e-mail at rramesh@berkeley.edu.

Ramesh is a professor at the University of California, Berkeley. He received his PhD degree from the University of California, Berkeley, in 1987. At the Lawrence Berkeley Laboratory, Ramesh carried out pio- neering research on hightemperature superconductors and codiscovered the $110 \mathrm{~K}$ superconducting phase in the bismuth cuprate system. While at Bellcore from 1989 to 1995 , he initiated research in several key areas, including ferroelectric nonvolatile memories. Ramesh joined the University of Maryland in 1995, where he was promoted to professor in 1999 and to Distinguished University Professor in 2003. His recent work has pioneered the resurgence of research activity in multifunctional materials. Ramesh has more than 350 publications, along with 18 patents issued, and 11 pending. With more than 14,000 citations, he ranks among the 


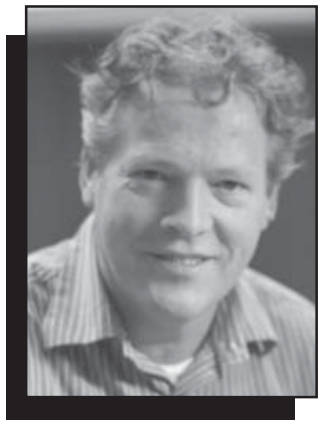

Dave H.A. Blank

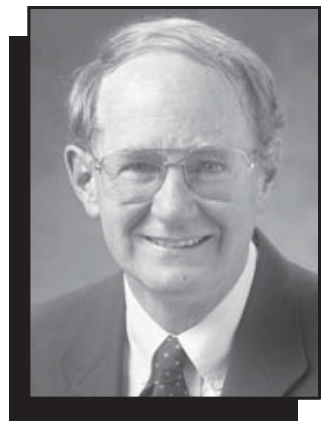

Scott A. Chambers

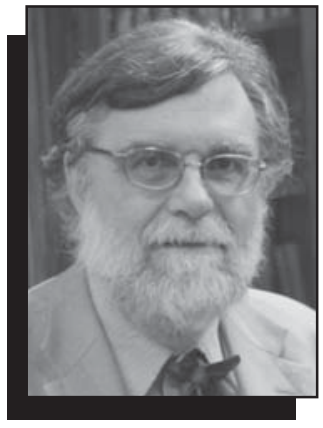

J. Michael D. Coey

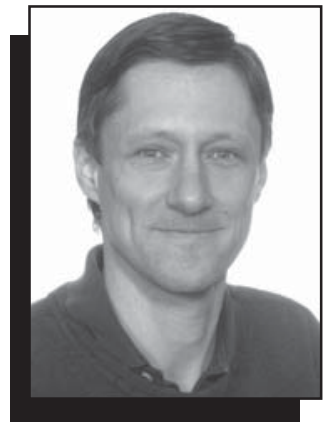

Elbio Dagotto

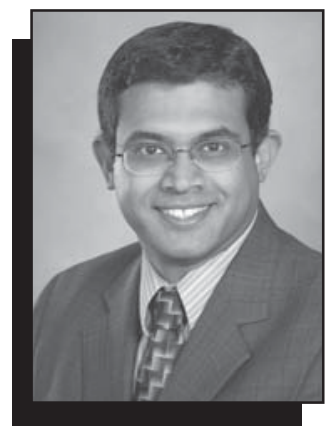

Suman Datta most cited researchers in physics. In 2000, Ramesh was awarded the Outstanding Achievement Award from the International Symposium on Integrated Ferroelectrics. He received the Humboldt Senior Scientist Prize from the Alexander von Humboldt Foundation for his pioneering work on the fundamental nanoscale science of size scaling in ferroelectric thin films; the A. James Clark College of Engineering Faculty Outstanding Research Award; and Fellowships to the American Physical Society (2001), and the American Association for the Advancement of Science (2005). In 2005, he received the APS David Adler Lectureship Award. The following year, he was awarded the Ikeda Lectureship in Japan and the Brahm Prakash Visiting Chair at the Indian Institute of Science.

\section{Darrell G. Schlom, Guest Editor for this issue of MRS Bulletin, can be reached by e-mail at schlom@ems.psu.edu. \\ Schlom is a professor in the Department of Materials Science and Engineering at Cornell University. He received his BS degree from the California Institute of}

Technology, and his MS and $\mathrm{PhD}$ degrees from Stanford University. After working as a postdoctoral researcher at IBM's research lab in Zurich, Switzerland, Schlom was on the faculty at Penn State University for 16 years. His research interests involve the growth and characterization of oxide thin films, including their integration with semiconductors. Schlom has published more than 300 papers and has seven patents. He has been awarded invention achievement awards by IBM and SRC; young investigator awards by ONR, NSF, and the American Association for Crystal Growth; an Alexander von Humboldt Research Fellowship; and the ASM International Bradley Stoughton Award for Young Teachers. In addition, Schlom is a fellow of the American Physical Society and the winner of this year's MRS Medal.

Dave H.A. Blank can be reached at MESA+

Institute for

Nanotechnology,

University of Twente, PO

Box 217, 7500 AE, The

Netherlands; tel. 31-53-

489-3121; and e-mail

d.h.a.blank@utwente.nl.

Blank is a professor in chemistry and materials science at University of Twente, The

Netherlands. He received his $\mathrm{PhD}$ degree in applied physics on high temperature superconductors. In 2007, Blank became the scientific director of MESA+ Institute for Nanotechnology, The

Netherlands. The major focus of his research is growth studies, deposition and structuring techniques, and properties of (artificial) complex materials, especially oxides. In his group, the first timeresolved RHEED-system is developed. In 2002,

Blank was awarded with the VICI laureate of the

Dutch Science

Foundation for his work on artificial materials for nanoscale devices. In 2004 , he became the flagship captain of nanoelectronic materials science of the

NANONED program (the Dutch initiative on nanotechnology).

\section{Scott A. Chambers can}

be reached at Pacific Northwest National Laboratory, PO Box 999, MS K8-87, Richland, WA 99352, USA; tel. 509-3716517; and e-mail sa.chambers@pnl.gov.

Chambers is a laboratory fellow and leader of the Oxide Epitaxy Group in the Fundamental and Computational Sciences Directorate at Pacific Northwest National
Laboratory in Richland, Washington. He also is an affiliate professor of materials science and engineering and an affiliate professor of chemistry at the University of Washington in Seattle. Chambers received his $\mathrm{PhD}$ degree in physical chemistry from Oregon State University in 1978. His principal research interests are in the epitaxial growth and properties of a variety of transition metal oxides. Chambers has authored or coauthored 200 journal articles, review articles, and book chapters, and is a fellow of the AVS.

\section{J. Michael D. Coey can} be reached at the School of Physics and CRANN, Trinity College, Dublin 2, Ireland; tel. 353-18961470; and e-mail jcoey@tcd.ie.

Coey is Deputy Director of CRANN, Ireland's Nanoscience Research Center, at Trinity College, Dublin. He obtained his $\mathrm{PhD}$ degree from the University of Manitoba in 1971. Coey has worked in magnetism ever since, in Grenoble, IBM Yorktown Heights, and in Dublin, where he holds the Erasmus Smith's Chair of Natural and Experimental Philosophy-a professorship of physics dating from 1724 . He has recently completed a new textbook on Magnetism and Magnetic Materials. A fellow of the Royal Society and foreign associate of the National Academy of Sciences, Michael Coey's current research interests include novel magnetic materials, spin electronics, magnetoelectrochemistry, and biomagnetism.

Elbio Dagotto can be reached at the Department of Physics, University of Tennessee, 1408 Circle Dr., Knoxville, TN 37996, USA; tel. 865974-3342, fax 865-974-

7843; and e-mail edagotto@utk.edu. Dagotto is

Distinguished Professor of Physics at the University of Tennessee and Distinguished Scientist at the Materials Science and Technology Division of Oak Ridge

National Laboratory. He received his $\mathrm{PhD}$ degree in physics in 1985 at the Instituto Balseiro, Bariloche, Argentina. Dagotto has been working mainly on computational studies of models for systems where strong correlations among the electrons play an important role. He is Fellow of the American Physical Society, member of the Solid State Sciences Committee of the National Academy of Sciences, and a former divisional editor of 


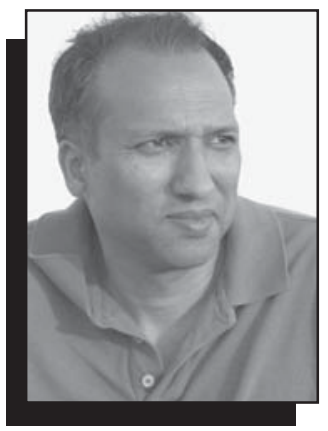

Supratik Guha

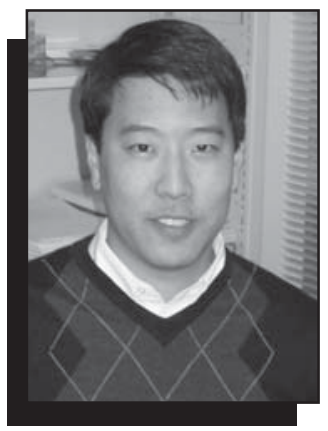

Harold Y. Hwang

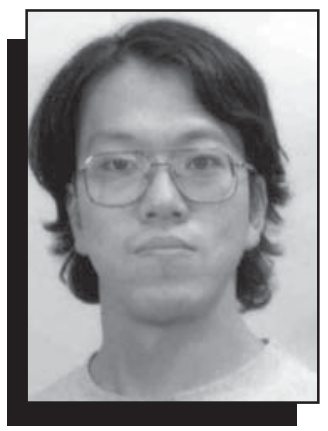

Toshio Kamiya

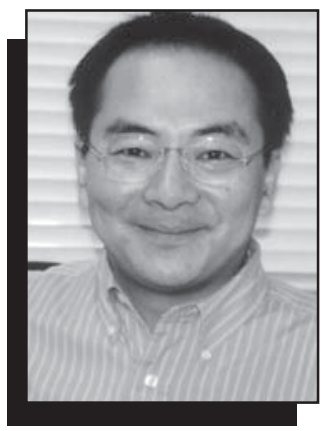

Masashi Kawasaki

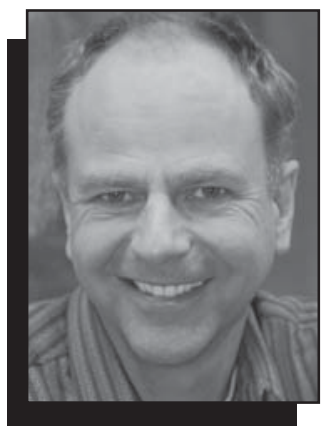

Jochen Mannhart
Physical Review Letters (1998-2002).

Suman Datta can be reached by e-mail at sdatta@engr.psu.edu.

Datta is an associate professor in the Department of Electrical Engineering at the Pennsylvania State University, where he currently holds the Monkowsky professorship for faculty career development. He received his bachelor's degree from the Indian Institute of Technology, Kanpur, India, and his $\mathrm{PhD}$ degree from the University of Cincinnati in 1995 and 1999 , respectively. Prior to joining Penn State, Datta was a principal engineer at Intel Corporation, where he was involved in the research and development of advanced logic transistors. His research interests involve simulation, design, fabrication, and characterization of ultralow power transistors and quantum devices. Datta has published more than 50 papers and holds 54 patents. He received the 2003 Intel Achievement Award and the 2002 Divisional Recognition Award for his inventions related to high performance high $-k /$ metal gate planar transistors and non-planar tri-gate transistors, respectively. In addition, Datta is a senior member of the IEEE

Electron Devices Society.

Supratik Guha can be reached by e-mail at guha@us.ibm.com.

Guha is a senior manager in the Physical Sciences Department at IBM Research, where he has been since 1995 . He received his $\mathrm{PhD}$ degree in materials science from the University of Southern California in 1991, and a BTech degree in metallurgical engineering from the Indian Institute of Technology, Kharagpur, in 1985. Guha also is an adjunct professor of materials science at Columbia University. From 1992 to 1995 , he worked at the $3 \mathrm{M}$ Corporate Research Labs in the II-VI semiconductor laser group. Guha is one of the early members of IBMs high$k$ research group and has led IBMs high- $k$ materials research program over the past decade. More recently, he has been responsible for establishing an active photovoltaics program at IBM. His research interests are in the area of new semiconductors and oxides for electronic and energy conversion applications. In addition, Guha is the author of more than 90 publications and has 27 patents.
Harold Y. Hwang can be reached at the University of Tokyo, Interdisciplinary Science Building 606, 5-1-5 Kashiwano-ha, Kashiwa-shi, Chiba 277-8561, Japan; and e-mail hyhwang@ k.u-tokyo.ac.jp.

Hwang is an associate professor in the Department of Advanced Materials Science and the Department of Applied Physics at the University of Tokyo, and with the Japan Science and Technology Agency. He received a BS degree in physics, BS and MS degrees in electrical engineering from the Massachusetts Institute of Technology (1993), and a $\mathrm{PhD}$ degree in physics from Princeton University (1997). Hwang was formerly a Member of the Technical Staff in the Materials Physics Research Department at Bell Laboratories, and a visiting professor at the Institute for Chemical Research, Kyoto University. His research interests include charge transport, spin and lattice dynamics, ordering transitions in transition metal oxides, and the atomic engineering of oxide heterointerfaces. Hwang received the 2005 MRS Outstanding Young Investigator Award, and was a meeting chair for the 2007 MRS Spring Meeting.
Toshio Kamiya can be reached by e-mail at kamiya.t.aa@m.titech.ac.jp.

Kamiya is an associate professor in the Materials and Structures

Laboratory at the Tokyo Institute of Technology. He started his research career as an assistant professor of Department of Inorganic Materials at Tokyo Institute of Technology in 1991, working on ferroelectric inorganic materials, and received his $\mathrm{PhD}$ degree in 1996. Kamiya moved to the Interdisciplinary Graduate School of Science and Engineering and worked with silicon thin films from 1996 to 2000. From 2000 to 2002, he spent two years as a visiting scholar at the Microelectronics Research Centre, Cavendish Laboratory, at the University of Cambridge, studying on single-electron transistors. Kamiya became an associate professor in 2002 and has since studied functional oxide materials. His field of research includes exploration, materials design, and device applications of new functional oxides. Kamiya has published approximately 200 scientific papers. In addition, he has received The Young Scientists' Prize by MEXT, The Advanced Technology Award by the Fuji-Sankei group, and The DV-X $\alpha$ Research Award for young scientists by the Society for Discrete Variational X $\alpha$.

Masashi Kawasaki can be reached by e-mail at kawasaki@imr. tohoku.ac.jp.

Kawasaki is a professor in the WPI Advanced Institute for Materials Research at Tohoku University, Japan. He received his $\mathrm{PhD}$ degree from the Department of Chemical Energy Engineering at the University of Tokyo in 1989, working on amorphous semiconductor superlattices and high $T_{c}$ superconducting films. Kawasaki spent two years at IBM Thomas J. Watson Research Center as a postdoctoral research fellow before he received a research associate position in 1991, and an associate professor position in 1997 at Tokyo Institute of Technology. He moved to Tohoku University as a full professor in 2001. Kawasaki is co-assigned as a team leader of Cross-Correlated Materials Research Group (CMRG) at RIKEN, chief innovation officer at COMET Corp., and a project leader of CREST of JST. His field of research includes optical, electronic, magnetic, and their mutual functionalities at the inter- 


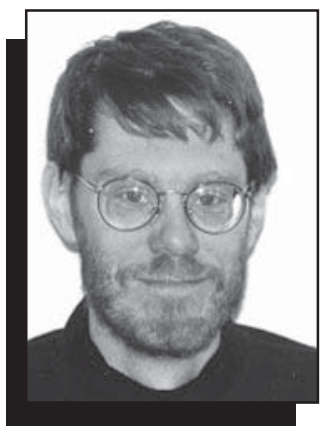

Andrew J. Millis

faces made of oxide semiconductors and correlated electron oxides. Kawasaki has published more than 400 scientific papers, from which more than 12,000 citations have been recorded ( $h$-index $=52$ ). In addition, he has received the IBM Japan Prize, the Marubun Scientific Award, the JSPS Prize, the Yamazaki-Teiichi Prize, and the CSJ Award for Creative Work.

Jochen Mannhart can be reached by e-mail at jochen.mannhart@physik .uni-augsburg.de.

Mannhart has been a Chaired Professor of Physics at the Center for Electronic Correlations and Magnetism at the University of Augsburg since 1996. He holds a diploma in physics from the University of Tübingen, Germany. Mannhart later received his $\mathrm{PhD}$ degree for imaging the Josephson current in tunnel junctions, also at Tübingen. Starting in 1987 , he worked as a visiting scientist at the IBM T.J. Watson Research Center in Yorktown

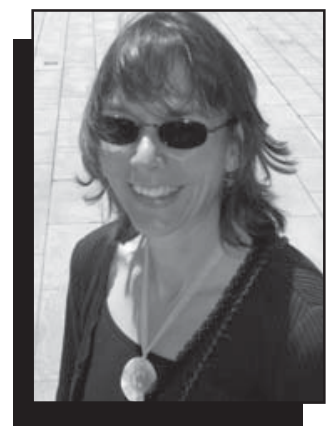

Nicola A. Spaldin

Heights, and as a research staff member at the IBM Zurich Research Laboratory in

Rüschlikon, Switzerland. Mannhart's interests focus on the properties of interfaces in complex electronic materials. The efforts of the various teams he has worked with have resulted in the development of bicrystal Josephson junctions and SQUIDs, the enhancement of grain boundary critical current density in high- $T_{\mathrm{c}}$ superconductors by grain alignment, and the fabrication of the first all-oxide FETs. Mannhart is a fellow of the Institute of Physics and of the American Physical Society. In 2008, he was awarded the Gottfried Wilhelm Leibniz-Preis of the German Science Foundation.

Andrew J. Millis can be reached by e-mail at millis@phys.columbia.edu.

Millis is a professor of physics and department chair at Columbia

University. Before joining Columbia, he was on the faculty of Rutgers and Johns Hopkins

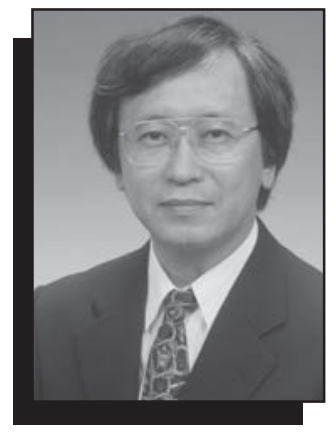

Yoshinori Tokura

Universities, and also worked for 10 years in the Theoretical Physics Research Department at AT\&T (later Lucent) Bell Labs. His research is in the area of theoretical condensed matter physics. Millis is known for his work on "heavy fermion" materials, on quantum criticality, on colossal magnetoresistance manganites, and on high-temperature superconductivity.

Nicola A. Spaldin can be reached at the Materials Department, University of California, Santa Barbara, CA 93106, USA; tel. 805-893-7920; fax 805-893-8797; and e-mail nicola@mrl. ucsb.edu.

Spaldin is a professor in the Materials Department at the University of California, Santa Barbara (UCSB). She joined UC Santa Barbara after receiving her PhD degree in chemistry from the University of California, Berkeley, and postdoctoral work in applied physics at Yale University. Spaldin's research focuses on the use of electronic structure

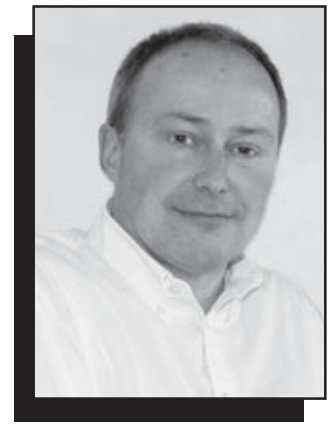

Jean-Marc Triscone

methods to design and understand novel multifunctional materials, particularly multiferroics. In addition, she is director of the UCSB-based National Science

Foundation International

Center for Materials

Research, and has an array of papers, invited talks, and awards.

Yoshinori Tokura can be reached at the

Department of Applied Physics, University of Tokyo, 7-3-1 Hongo, Bunkyo-ku, Tokyo 1138656, Japan; tel. 81-3-58416870; fax 81-3-5841-6839; and e-mail tokura@ ap.t.u-tokyo.ac.jp.

Tokura is a professor of applied physics at the University of Tokyo.

Tokura also is group director at the Advanced Science Institute, RIKEN, and project director of the ERATO Multiferroics Project, JST. He received his doctoral degree in 1981 at the University of Tokyo. Tokura has been working mainly on materials physics of correlated-electron systems. He received the Nishina Memorial Prize (1991), the Bernd Matthias Prize
(1992), the Asahi Prize (2002), and the James C. McGroddy Prize (2005), among others, for his discoveries of new correlated-electron materials and phenomena.

Jean-Marc Triscone can be reached at DPMC, University of Geneva, 24 quai E.-Ansermet, 1211 Geneva 4, Switzerland; tel. 41-22-379-6651; and e-mail jean-marc.triscone@ physics.unige.ch.

Triscone is a professor of physics in the Condensed Matter Physics Department at the University of Geneva, leading a group working on nanoscale studies of ferroelectrics, field effects in correlated oxide systems, and oxide interface physics.

Triscone obtained his PhD degree in 1987 with professor Oystein Fischer, studying metallic superconducting superlattices. In 1990, 1991, and 1995, he was a visiting scientist at Stanford University. Since 1996, Triscone has been a full professor at Geneva. Between 2001 and 2007, he was the deputy-director of the National Center of Competence in Research MaNEP. Since July 2007,

Triscone has been the Dean of the Faculty of Sciences at Geneva. With D. Blank and

J. Mannhart, he is leading the European Science Foundation network Thin Films For Novel Oxide Devices (THIOX). In addition, Triscone has been an APS fellow since 2006.

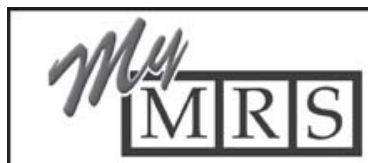

This new feature of the MRS Web site allows you to personalize the information you receive from usidentify your particular areas of interest, register for/manage your online newsletters and alerts, change your password, and update your contact information. 